\title{
Dual Meissner effect and non-Abelian dual superconductivity in SU(3) Yang-Mills theory
}

\author{
Akihiro Shibata* \\ High Energy Accelerator Research Organization (KEK) \\ E-mail: Akihiro.Shibata@kek.jp \\ Kei-Ichi Kondo \\ Department of Physics, Graduate School of Science, Chiba University, Chiba 263-8522, Japan \\ E-mail: kondokefaculty.chiba-u.jp
}

\section{Toru Shinohara}

Graduate School of Science, Chiba University, Chiba 263-8522, Japan

E-mail: sinohara@graduate.chiba-u.jp

The dual Meissner effect is the promising mechanism for quark confinement. We have proposed a new formulation of SU(N) Yang-Mills (YM) theory on a lattice, which can extract the dominant mode for quark confinement in the gauge independent manner. In the last lattice conference, we have demonstrated by measuring the string tension from the Wilson loop average in the SU(3) YM theory that the restricted non-Abelian variable and the extracted non-Abelian magnetic monopoles play the dominant role in confinement of fundamental quarks (dominance in the string tension), in marked contrast to the Abelian projection. In this talk, we focus on the dual Meissner effect in SU(3) YM theory, which is examined by measuring the distribution of chromo-electric field strength created by a static quark-antiquark pair. We apply the new lattice formulation, and examine whether or not the non-Abelian dual superconductivity claimed by us is indeed a mechanism of quark confinement. We present a preliminary result of the direct evidence for the non-abelian dual Meissner effect, that is to say, restricted U(2)-field part of the flux tube plays the dominant role in the quark-antiquark potential.

XXIX International Symposium on Lattice Field Theory

July 10 - 162011

Squaw Valley, Lake Tahoe, California

*Speaker. 


\section{Introduction}

Quark confinement follows from the area law of the Wilson loop average. The dual Meissner effect is the promising mechanism for quark confinement [1]. Based on the Abelian projection there are many numerical analyses which support the dual superconductivity picture, such as Abelian dominance [2], magnetic monopole dominance [3] [4], and center vortex dominance [5] in the string tension. However, these results are obtained only in special gauges such as the maximal Abelian (MA) gauge and the Laplacian Abelian gauge. Such gauge fixings also cause the problem of the color symmetry (global symmetry) breaking.

To overcome these problems, we have presented a new lattice formulation of $S U(N)$ Yang-Mills (YM) theory[7][10], that gives the decomposition of the gauge link variable suited for extracting the dominant mode for quark confinement. In the case of the $S U(2)$ YM theory, the decomposition of the gauge link variable is given by a compact representation of Cho-Duan-Ge-Faddeev-Niemi (CDGFN) decomposition[6] on a lattice.[8][9] For the $S U(N)$ YM theory, the new formula for the decomposition of the gauge link variable is constructed as an extension of the $S U(2)$ case. There are several possibilities of decomposition an extension of the $S U(2)$ case. There are several possibilities of decomposition corresponding to the stability subgroup $\tilde{H}$ of gauge symmetry group $G$, while there is the unique option of $\tilde{H}=U(1)$ in the $S U(2)$. For the case of $G=S U(3)$, there are two possibility which we call the maximal option and the minimal option. The maximal option is obtained for the stability group $\tilde{H}=U(1) \times U(1)$, which is the gauge invariant version of the Abelian projection in the maximal Abelian (MA) gauge [11][12]. The minimal one is obtained for $\tilde{H}=U(2) \cong S U(2) \times U(1)$, suitable for the Wilson loop in the fundamental representation [18]. We have demonstrated the gauge independent (invariant) restricted $U(2)$-dominance, (or conventionally called "Abelian" dominance), $\left(\sigma_{V} / \sigma_{\text {full }}=93 \pm 16 \%\right)$ where the decomposed $V$-field (restricted $\mathrm{U}(2)$ field) reproduced the string tension of original YM field, and the gauge independent non-Abelian magnetic monopole dominance $\left(\sigma_{m o n} / \sigma_{V}=94 \pm 9 \%\right)$, where the string tension was reproduced by only the (non-Abelian) magnetic monopole part extracted from the restricted U(2) field (see Figure 1) [14][13] [15][17].

To establish the dual superconductivity picture, the dual Meissner effect in Yang-Mills theory must be examined by measuring the distribution of chromo-electric field strength or color flux created by a static quark-antiquark pair. There are many works on color flux for the Yang-Mills field by using Wilson line/loop operator[20] [22] [21] [23] [24]. However, there is no direct measurement of the dual Meissner effect for the $S U(3)$ YM theory in the gauge independent (invariant) way. In this talk, we focus on the dual Meissner effect. Applying our new formulation to the $S U$ (3) YM theory, we measure the distribution of the extracted chromo-electric field strength which is created by a static quark-antiquark pair. Then, we investigate whether or not the non-Abelian dual superconductivity claimed by us is indeed a mechanism of quark confinement in $S U(3)$ YM theory.

\section{Extracting the dominant mode for dual super conductivity}

We briefly review a new formulation of the lattice YM theory, which extracts non-Abelian dual superconductivity mode for $S U(3)$ YM theory.[17, 15] In this talk, we use the minimal option, since we consider the quark confinement in the fundamental representation. Let us consider the decomposition of link variables, $U_{x, \mu}=X_{x, \mu} V_{x, \mu}$, where $V_{x, \mu}$ could be the dominant mode for quark confinement, and $X_{x, \mu}$ the remainder part. The YM field and the decomposed new-variables are transformed by full 

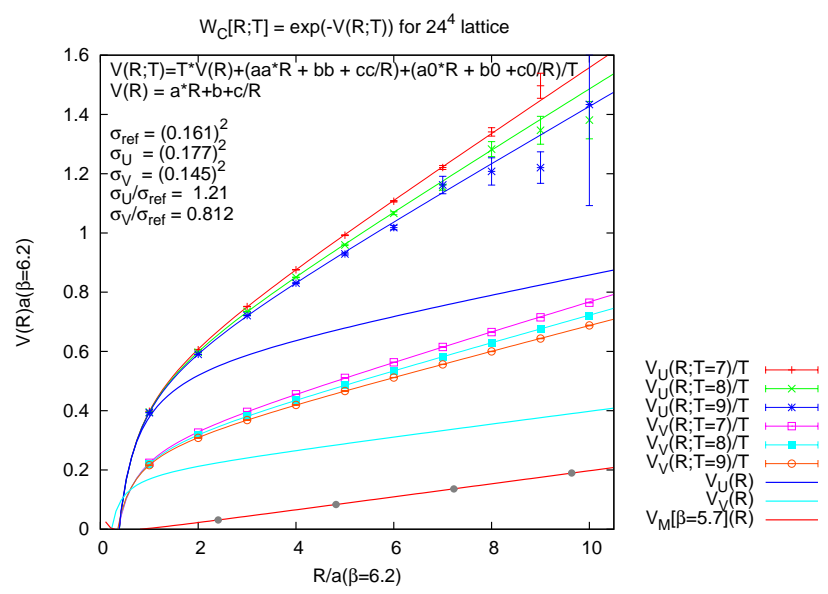

Figure 1: The quark-antiquark potential calculated using three types of sources shown from top to bottom: (a) the original YM field $(U)$, (b) the restricted $U(2)$ field ( $V$-field) and (c) the non-abelian magnetic monopole.

$S U$ (3) gauge transformation $\Omega_{x}$ such that $V_{x, \mu}$ is transformed as the gauge link variable and $X_{x, \mu}$ as the site available:

$$
\begin{aligned}
U_{x, \mu} \longrightarrow U_{x, v}^{\prime} & =\Omega_{x} U_{x, \mu} \Omega_{x+\mu}^{\dagger}, \\
V_{x, \mu} \longrightarrow V_{x, v}^{\prime} & =\Omega_{x} V_{x, \mu} \Omega_{x+\mu}^{\dagger}, X_{x, \mu} \longrightarrow X_{x, v}^{\prime}=\Omega_{x} X_{x, \mu} \Omega_{x}^{\dagger} .
\end{aligned}
$$

The decomposition is determined by the defining equation

$$
\begin{aligned}
& D_{\mu}^{\varepsilon}[V] \mathbf{h}_{x}:=\frac{1}{\varepsilon}\left[V_{x, \mu} \mathbf{h}_{x+\mu}-\mathbf{h}_{x} V_{x, \mu}\right]=0, \\
& g_{x}:=e^{i 2 \pi q / N} \exp \left(-i a_{x}^{0} \mathbf{h}_{x}-i \sum_{j=1}^{3} a_{x}^{(j)} \mathbf{u}_{x}^{(i)}\right)=1,
\end{aligned}
$$

where $\mathbf{h}_{x}$ is a introduced color field $\mathbf{h}_{x}=\xi\left(\lambda^{8} / 2\right) \xi^{\dagger} \in[S U(3) / U(2)]$ with $\lambda^{8}$ being the Gell-Mann matrix and $\xi$ the $S U(3)$ gauge element. The variable $g_{x}$ is undetermined parameter from Eq.(2.2a), $\mathbf{u}_{x}^{(j)}$ 's are $s u(2)$-Lie algebra values, and $q_{x}$ an integer value $0,1,2, \ldots, N-1$, respectively. These defining equations can be solved exactly [16], and the solution is given by

$$
\begin{aligned}
L_{x, \mu} & =\frac{N^{2}-2 N+2}{N} \mathbf{1}+(N-2) \sqrt{\frac{2(N-1)}{N}}\left(\mathbf{h}_{x}+U_{x, \mu} \mathbf{h}_{x+\mu} U_{x, \mu}^{\dagger}\right), \\
& +4(N-1) \mathbf{h}_{x} U_{x, \mu} \mathbf{h}_{x+\mu} U_{x, \mu}^{\dagger}, \\
\widehat{L}_{x, \mu} & =\left(L_{x, \mu} L_{x, \mu}^{\dagger}\right)^{-1 / 2} L_{x, \mu}, \\
X_{x, \mu} & =\widehat{L}_{x, \mu}^{\dagger} \operatorname{det}\left(\widehat{L}_{x, \mu}\right)^{1 / N} g_{x}^{-1}, \quad V_{x, \mu}=X_{x, \mu}^{\dagger} U_{x, \mu}=g_{x} \widehat{L}_{x, \mu} U_{x, \mu} .
\end{aligned}
$$

Note that the above defining equations correspond to the continuum version: $D_{\mu}[\mathscr{V}] \mathbf{h}(x)=0$ and $\operatorname{tr}\left(\mathbf{h}(x) \mathscr{X}_{\mu}(x)\right)=0$, respectively. In the naive continuum limit, we have the corresponding decomposition, $\mathbf{A}_{\mu}(x)=\mathbf{V}_{\mu}(x)+\mathbf{X}_{\mu}(x)$ as

$$
\begin{aligned}
& \mathbf{V}_{\mu}(x)=\mathbf{A}_{\mu}(x)-\frac{2(N-1)}{N}\left[\mathbf{h}(x),\left[\mathbf{h}(x), \mathbf{A}_{\mu}(x)\right]\right]-i g^{-1} \frac{2(N-1)}{N}\left[\partial_{\mu} \mathbf{h}(x), \mathbf{h}(x)\right], \\
& \mathbf{X}_{\mu}(x)=\frac{2(N-1)}{N}\left[\mathbf{h}(x),\left[\mathbf{h}(x), \mathbf{A}_{\mu}(x)\right]\right]+i g^{-1} \frac{2(N-1)}{N}\left[\partial_{\mu} \mathbf{h}(x), \mathbf{h}(x)\right] .
\end{aligned}
$$


To determine the decomposition, we need to determine the color field. Here, we use the reduction condition to minimize the functional

$$
F_{\text {red }}\left[\mathbf{h}_{x}\right]=\sum_{x, \mu} \operatorname{tr}\left\{\left(D_{\mu}^{\varepsilon}\left[U_{x, \mu}\right] \mathbf{h}_{x}\right)^{\dagger}\left(D_{\mu}^{\varepsilon}\left[U_{x, \mu}\right] \mathbf{h}_{x}\right)\right\}
$$

which is equivalent to solving the ground state of a spin glass.

From the non-Abelian Stokes' theorem[18][19] and the Hodge decomposition of the field strength $\mathscr{F}_{\mu \nu}[\mathbf{V}]$, we obtain the magnetic part (magnetic monopole current) in the gauge independent way. Therefore, the gauge invariant magnetic monopole is defined by using the $V$-field as

$$
\begin{aligned}
& V_{x, \mu} V_{x+\mu, \mu} V_{x+v, \mu}^{\dagger} V_{x, v}^{\dagger}=\exp \left(-i g \mathscr{F}_{\mu \nu}[\mathbf{V}]\right)=\exp \left(-i g \Theta_{\mu \nu}^{8} \mathbf{h}(x)\right) \\
& \Theta_{\mu v}^{8}:=-\arg \operatorname{Tr}\left[\left(\frac{1}{3} \mathbf{1}-\frac{2}{\sqrt{3}} \mathbf{h}_{x}\right) V_{x, \mu} V_{x+\mu, \mu} V_{x+v, \mu}^{\dagger} V_{x, v}^{\dagger}\right], \\
& k_{\mu}=2 \pi n_{\mu}:=\frac{1}{2} \varepsilon_{\mu v \alpha \beta} \partial_{\nu} \Theta_{\alpha \beta}^{8} .
\end{aligned}
$$

Note that the the current $k_{\mu}$ is the non-Abelian magnetic monopole current, since $V_{x, \mu}$ is the decomposed restricted $U(2)$ filed.

\section{Measurements of color flux}

We investigate the color flux produced by a quark-antiquark pair, see the left panel of Fig. 2 . In order to explore the color flux in the gauge invariant way, we use the connected correlator of the Wilson line [25] (see the right panel of Fig.2),

$$
\rho_{W}:=\frac{\left\langle\operatorname{tr}\left(U_{p} L^{\dagger} W L\right)\right\rangle}{\langle\operatorname{tr}(W)\rangle}-\frac{1}{N} \frac{\left\langle\operatorname{tr}\left(U_{p}\right) \operatorname{tr}(W)\right\rangle}{\langle\operatorname{tr}(W)\rangle},
$$

where $W$ represents the Wilson loop as the quark and anti-quark source term, $U_{p}$ a plaquette variable as the probe operator to measure field strength, $L$ the Wilson line connecting the source $W$ and probe $U_{p}$, and $N$ the number of color $(N=3)$. The symbol $\langle\mathscr{O}\rangle$ denotes the average of the operator $\mathscr{O}$ in the space and the ensemble of the configurations. Note that this is sensitive to the field strength rather than the disconnected one. Indeed, in the naive continuum limit, the connected correlator $\rho_{W}$ is given by

$$
\rho_{W} \stackrel{\varepsilon \rightarrow 0}{\simeq} g \varepsilon^{2}\left\langle\mathscr{F}_{\mu \nu}\right\rangle_{q \bar{q}}:=\frac{\left\langle\operatorname{tr}\left(g \varepsilon^{2} \mathscr{F}_{\mu v} L^{\dagger} W L\right)\right\rangle}{\langle\operatorname{tr}(W)\rangle}+O\left(\varepsilon^{4}\right)
$$

while the disconnected one is given by

$$
\rho_{W}^{\prime}:=\frac{\left\langle\operatorname{tr}(W) \operatorname{tr}\left(U_{p}\right)\right\rangle}{\langle\operatorname{tr}(W)\rangle}-\left\langle\operatorname{tr}\left(U_{p}\right)\right\rangle \stackrel{\varepsilon \rightarrow^{0}}{\simeq} g \varepsilon^{4}\left[\left\langle\mathscr{F}_{\mu v}^{2}\right\rangle_{q \bar{q}}-\left\langle\mathscr{F}_{\mu v}^{2}\right\rangle_{0}\right]
$$

Thus, the color filed strength is given by $F_{\mu v}=\sqrt{\frac{\beta}{2 N}} \rho_{W}$.

To investigate the non-Abelian dual Meissner effect as the mechanism of quark confinement, we measure correlators of the restricted $U(2)$ field, $V_{x, \mu}$, in place of the original YM filed. Note again that this restricted $U(2)$-field and the non-Abelian magnetic monopole extracted from it reproduce the string tension in the quark-antiquark potential. (see Fig. 1) 

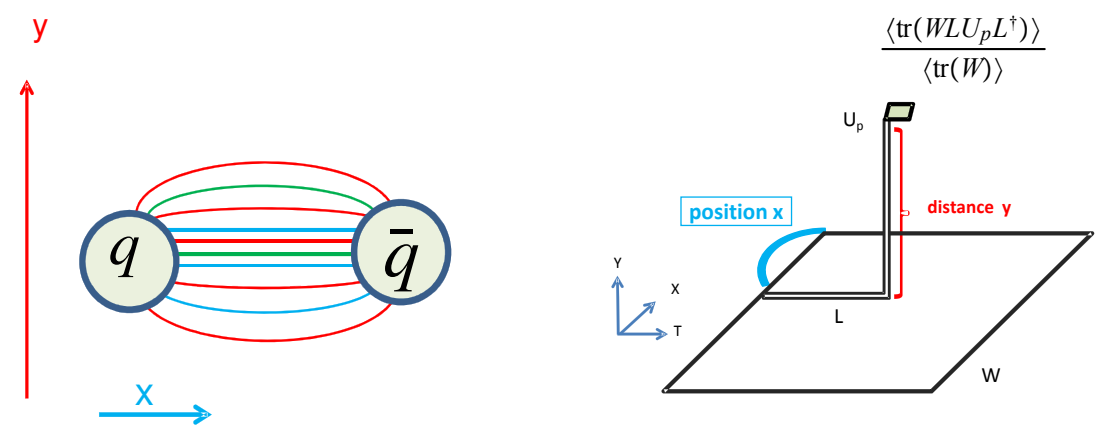

Figure 2: (Left) The measurement setup of the color flux produced by a quark-antiquark pair. (Right) The connected correlator between a plaquette and the Wilson loop.
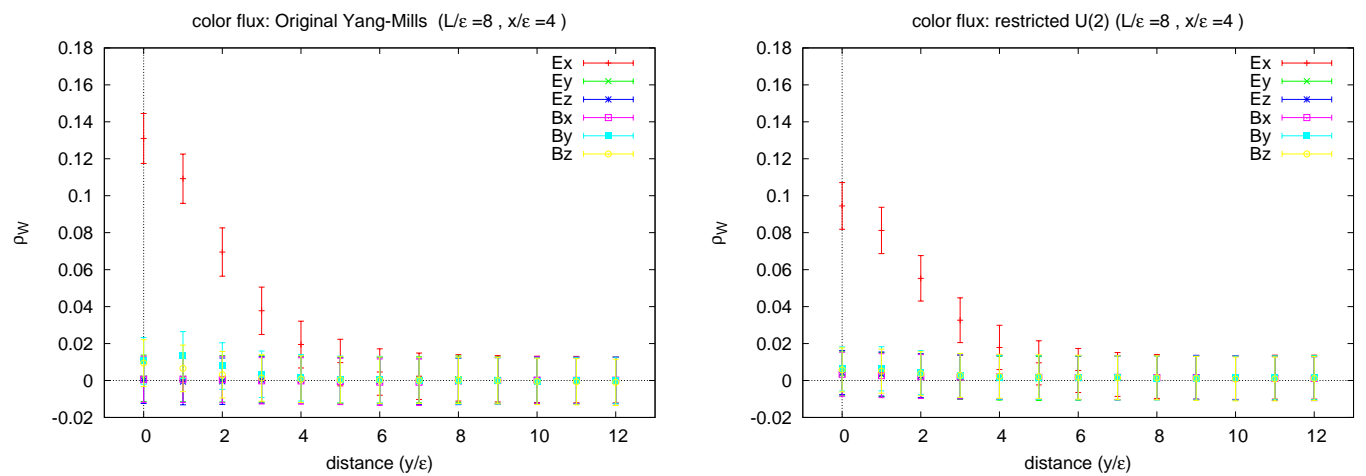

Figure 3: Measurement of $E_{x}$ component of the color flux in the x-y plane. (Left panel) The $E_{x}$ for the $S U(3)$ YM field (Right panel) $E_{x}$ component for the restricted $U(2)$ field.

We generate gauge configurations using the standard Wilson action on a $24^{4}$ lattice with $\beta=6$. The gauge link decomposition is obtained by the formula given in the previous section, i.e., the color field configuration is obtained by solving the reduction condition of minimizing the functional eq(2.5), and the decomposition is obtained by using the formula eq(2.3). In the measurement, we apply the APE smearing method to reduce the noise.

The left panel of Fig. 3 shows the result of measurements of the color flux obtained from the original YM field. Here the quark and antiquark source is introduce as $8 \times 8$ Wilson loop $(W)$ in the $\mathrm{X}$-T plane, and the probe $\left(U_{p}\right)$ is set at the center of Wilson loop and moved along the Y-direction. (see Fig. 2). The $E_{x}$ component only has non-zero value, and it decreases quickly as away from the Wilson loop. The right panel of Fig. 3 shows the color flux obtained from the restricted $U(2)$-field, where the gauge link variable $V_{x, \mu}$ is used in place of the YM field $U_{x, \mu}$ in eq(3.7). The $E_{x}$ component only has non-zero value as well as the original YM filed case, and color flux is detected only along the Wilson loop. Then, to investigate the profile of the color flux tube we explore the distribution of color flux in the dimensional plane. Fig. 4 shows the magnitude of $E_{x}$ of the color flux in the X-Y plane. The quark and antiquark source is introduced as $9 \times 11$ Wilson loop in the X-T plane. The probe is displaced on the $\mathrm{X}-\mathrm{Y}$ plane at the midpoint of the Wilson loop in the T-direction. The position of the 

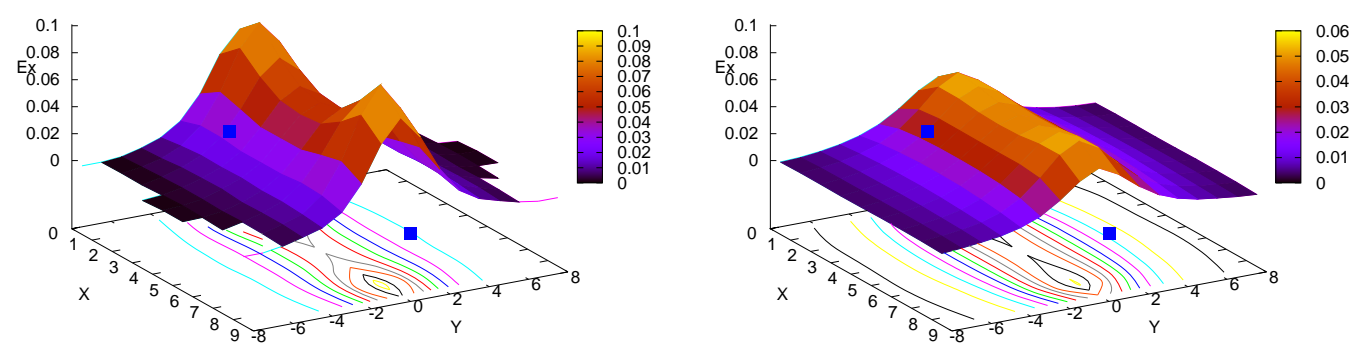

Figure 4: Measurement of color flux. (Left panel) $E_{x}$ of the $S U$ (3) YM field. (Right panel) $E_{x}$ component of the restricted $U(2)$ field.

quark and antiquark is marked by the solid blue box. The magnitude is shown by the height of the 3D plot and also the contour in the bottom plane. The left panel shows the color flux of the YM filed, and the right panel of the restricted $U(2)$-field. The figures show that the color flux of the restricted $U(2)$-field or $\mathbf{V}_{\mu}(x)$ component, is narrowed down to the tube. While, as for the original YM field the color flux of the $\mathbf{X}_{\mu}(x)$ component could be detected near the quark and antiquark sources.

\section{Summary and outlook}

We have studied the dual Meissner effect for $S U$ (3) YM theory by using our new formulation of YM theory on a lattice. We have extracted the restricted $U(2)$ field from the YM field which play a dominant role in confinement of quark (fermion in the fundamental representation), i.e., the restricted $U(2)$ dominance and the non-Abelian magnetic monopole dominance in the string tension. By measuring the color flux for both the original YM field and the restricted $U(2)$ field, we have investigated the dual Messier effect of $S U$ (3) YM theory. We have found that the electric part of the color flux is narrowed down in both cases, and the restricted $U(2)$ field part is dominant in the long distance region. We have found the tube-shaped flux of the electric part of the restricted $U(2)$ color field, which is a direct evidence of the non-Abelian dual superconductivity.

To confirm the non-Abelian dual superconductivity picture, we need further study on the dual Meissner effect of $S U$ (3) YM theory, e.g., determination of the type of dual super conductor, measurement of the penetrating depth, induced magnetic current around color flux due to the magnetic monopole condensation, and so on.

\section{Acknowledgement}

This work is supported by Grant-in-Aid for Scientific Research (C) 21540256 from Japan Society for the Promotion Science (JSPS), and also in part by JSPS Grant-in-Aid for Scientific Research (S) 22224003. The numerical calculations are supported by the Large Scale Simulation Program No.09/10-19 (FY2009-2010) and No.10-13 (FY2010) of High Energy Accelerator Research Organization (KEK). 


\section{References}

[1] Y. Nambu, Phys. Rev. D10, 4262(1974); G. 't Hooft, in: High Energy Physics, edited by A.; Zichichi (Editorice Compositori, Bologna, 1975).; S. Mandelstam, Phys. Report 23, 245(1976).; A.M. Polyakov, Nucl. Phys. B120, 429(1977).

[2] T. Suzuki and I. Yotsuyanagi, Phys. Rev. D42 4275 (1990)

[3] J.D.Stack, S.D. Neiman and R.Wensley, Phys. Rev. D50 3399 (1994)

[4] H. Shiba and T. Suzuki, Phys. Lett. B351 519 (1995)

[5] J. Greensite, Prog. Part. Nucl. Phys. 511 (2003)

[6] Y.M. Cho, Phys. Rev. D 21, 1080 (1980). Phys. Rev. D 23, 2415 (1981); L. Faddeev and A.J. Niemi, Phys. Rev. Lett. 82, 1624 (1999); S.V. Shabanov, Phys. Lett. B 458, 322 (1999). Phys. Lett. B 463, 263 (1999).

[7] K.-I. Kondo, T. Murakami and T. Shinohara, Eur. Phys. J. C 42, 475 (2005); K.-I. Kondo, T. Murakami and T. Shinohara, Prog. Theor. Phys. 115, 201 (2006).

[8] S. Ito, S. Kato, K.-I. Kondo, A. Shibata, T. Shinohara, Phys.Lett. B645 67-74 (2007)

[9] A. Shibata, S. Kato, K.-I. Kondo, T. Murakami, T. Shinohara, S. Ito, Phys.Lett. B653 101-108 (2007)

[10] K.-I. Kondo, T. Shinohara and T. Murakami, Prog.Theor. Phys. 120, 1 (2008); K.-I. Kondo, A.Shibata, T. Shinohara, T. Murakami, S. Kato and S. Ito, Phys. Lett. B669, 107 (2008)

[11] Akihiro Shibata, Seiko Kato,Kei-Ichi Kondo, Toru Shinohara and Shoichi Ito, CHIBA-EP-166, KEK-PREPRINT-2007-50, POS(LATTICE2007) 331, arXiv:0710.3221 [hep-lat]

[12] Akihiro Shibata, Seiko Kato,Keiichi Kondo, Toru Shinohara and Shoichi Ito, KEK-PREPRINT-2008-36, CHIBA-EP-173, 56 [hep-lat], PoS(LATTICE 2008) 268

[13] S. Kato, K-I. Kondo, A. Shibata and T. Shinohara, PoS(LAT2009) 228.

[14] Akihiro Shibata, Kei-Ichi Kondo, Seikou Kato, Shoichi Ito, Toru Shinohara, Nobuyui Fukui, PoS LAT2009 (2009) 232, arXiv:0911.4533 [hep-lat].

[15] A. Shibata, K-I. Kondo,S. Kato and T. Shinohara, PoS(Lattice 2010)286

[16] A. Shibata, K.-I. Kondo and T. Shinohara, Phys.Lett.B691:91-98,2010, arXiv:0911.5294[hep-lat]

[17] Kei-Ichi Kondo, Akihiro Shibata, Toru Shinohara, Seikou Kato, Phys.Rev. D83 (2011) 114016

[18] K.-I. Kondo, Phys.Rev.D77 085029 (2008)

[19] K.-I. Kondo and A. Shibata, CHIBA-EP-170, KEK-PREPRINT-2007-73, arXiv:0801.4203[hep-th]

[20] Mario Salvatore Cardaci, Paolo Cea, Leonardo Cosmai, Rossella Falcone and Alessandro Papa, Phys.Rev.D83:014502,2011

[21] N. Cardoso, M. Cardoso, P. Bicudo, arXiv:1107.1355 [hep-lat] (also lattice2011)

[22] Ahmed S. Bakry, Derek B. Leinweber, Anthony G. Williams, e-Print: arXiv:1107.0150 [hep-lat]

[23] Pedro Bicudo,Marco Cardoso, Nuno Cardoso, PoS LATTICE2010:268, 2010.

[24] Paolo Cea, Leonardo Cosmai, Phys.Rev.D52:5152-5164,1995.

[25] Adriano Di Giacomo et.al, Phys.Lett.B236:199,1990 Nucl.Phys.B347:441-460,1990 